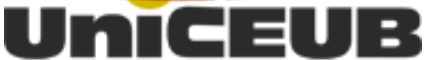 \\ Centro Unıversıtárıo de Brasílıa \\ CENTRO UNIVERSITÁRIO DE BRASÍLIA - UniCEUB PROGRAMA DE INICIAÇÃO CIENTÍFICA
}

WESLEY HENRIQUE SEIXAS MARTINS

O CUSTO DA FRATURA DE QUADRIL EM IDOSOS ANÁLISE TEMPORAL DO IMPACTO ECONÔMICO PARA O SISTEMA ÚNICO DE SAÚDE NO DISTRITO FEDERAL

BRASÍLIA

2018 


\section{Unce =Us \\ Centro Unıversıtárıo de Brasílıa}

\section{WESLEY HENRIQUE SEIXAS MARTINS}

\section{O CUSTO DA FRATURA DE QUADRIL EM IDOSOS ANÁLISE TEMPORAL DO IMPACTO ECONÔMICO PARA O SISTEMA ÚNICO DE SAÚDE NO DISTRITO FEDERAL}

Relatório final de pesquisa de Iniciação Científica apresentado à Assessoria de Pós-Graduação e Pesquisa.

Orientação: Viviane Cristina Uliana Peterle

BRASÍLIA 


\section{DEDICATÓRIA}

Dedico esse projeto aos meus pais que acreditaram no meu potencial e não mediram esforços para financiar a busca dos meus sonhos, sem eles essa caminhada não seria possível. 


\section{AGRADECIMENTOS}

Agradeço em primeiro lugar a Deus que iluminou meu caminho durante esse e outros projetos da minha vida. Agradeço também a minha orientadora, Dra. Viviane, por compartilhar comigo um pouco de seu conhecimento e por todo auxílio durante esse projeto. 
"A verdadeira viagem de descobrimento não consiste em procurar novas paisagens, mas em ter novos olhos". (Marcel Proust) 


\title{
O CUSTO DA FRATURA DE QUADRIL EM IDOSOS ANÁLISE TEMPORAL DO IMPACTO ECONÔMICO PARA O SISTEMA ÚNICO DE SAÚDE NO DISTRITO FEDERAL
}

\author{
Wesley Henrique Seixas Martins \\ Henrique.martins@sempreceub.com \\ Viviane Cristina Uliana Peterle \\ Viviane.peterle@uniceub.br
}

Objetivo: delinear o perfil de custos relacionados a fratura de quadril no Distrito Federal, enfatizando o crescimento acelerado em número de idosos no Brasil nos últimos anos o que poderá implicar mais internações por acometimento do quadril, mostrando a importância da prevenção desse tipo de fratura. Métodos: refere-se a um estudo descritivo a partir de dados obtidos no Sistema de Informação Hospitalares do Sistema único de Saúde (SIH-SUS). O SHI-SUS faz parte de um conjunto de sistemas do departamento de informática do SUS, o DATASUS, que tem como objetivo disponibilizar informações que servem para análises mais fidedignas da situação sanitária da população. Fez-se a coleta de dados de morbidade hospitalar devido a fratura de fêmur em pacientes acima de 60 anos. Resultados: No Distrito Federal ocorreram 3.721 internações por fratura de quadril em idosos no período de 2008 a 2016, o que corresponde a cerca de $1 \%$ das internações por fratura de quadril no Brasil. Essas internações totalizaram um custo de $R \$ 8.180 .600,70 \mathrm{mi}$, com média de $\mathrm{R} \$ 908.955,63$ por ano. Cada internação teve um valor médio de $R \$ 2.198,50$. Foi constatado um aumento de $42 \%$ em relação ao gasto do Sistema Único de Saúde com essa fratura entre os anos de 2008 a 2016. Cerca de 65\% dos pacientes eram do sexo feminino. A faixa etária acima dos 80 anos foi a faixa etária mais acometida, com $41 \%$ das internações. Conclusão: nosso estudo mostra um impacto econômico significante referente aos custos de internação por fratura de quadril já que esse tipo de fratura requer cuidados hospitalares de média e longa duração, o que leva a um custo elevado para o Sistema Único de Saúde. Além disso, há uma expetativa que os custos com esse tipo de fratura aumentem tendo em vista o crescimento do número de idosos, principal grupo social acometido por essa fratura.

Palavras-Chave: Fratura de quadril. Morbidade. Custos econômicos. Idoso. 


\section{LISTAS}

1- Custo em reais por ano no Distrito Federal ................................................. 5

2- Custo em reais por sexo no Distrito Federal ............................................ 6

3- Custo em reais por faixa etária no Distrito Federal ....................................... 6 
SUMÁRIO

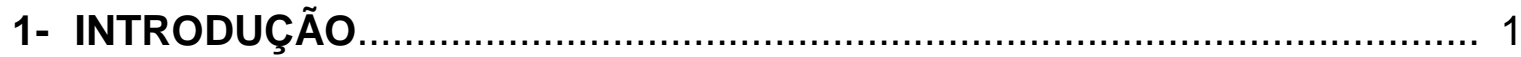

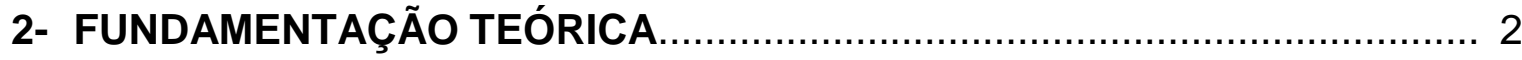

3- METODOLOGIA

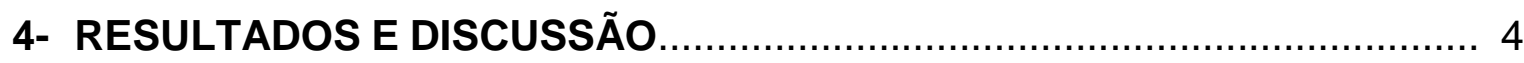

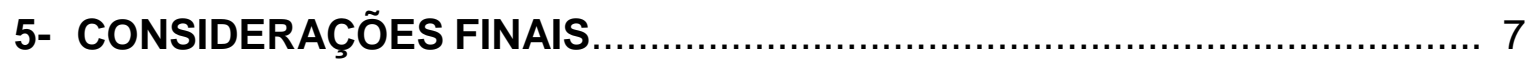

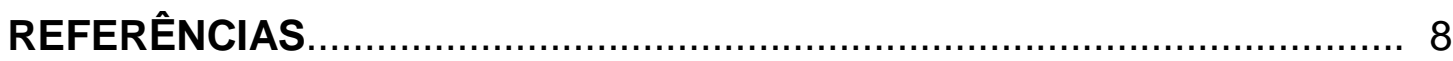




\section{Introdução:}

Esse estudo trata sobre o custo relacionado ao tratamento da fratura de quadril em pacientes acima dos 60 anos internados em hospitais do Distrito Federal. As fraturas de quadril requerem cuidados hospitalares e estão associadas a riscos elevados de morbidade, mortalidade e re-hospitalização. Esses cuidados demandam custos econômicos ao governo e com o aumento da expectativa de vida da população brasileira, espera-se um crescimento do número de internações por fraturas de quadril em idosos, elevando assim as despesas do Sistema Único de Saúde (SUS).

Graças aos avanços da medicina e as melhorias sanitárias vivenciadas pela população brasileira nas últimas décadas, a expectativa média de vida do brasileiro, que em 1940 era de 45,5 anos de idade, passou para 72,7 anos em 2008, um acréscimo de mais 27,2 anos de vida. Segundo a projeção do IBGE, o pais continuará com um padrão ascendente de expectativa de vida, alcançando em 2050 o patamar de 81,29 anos. (ARNDT, 2009)

Com o avançar da idade cresce o número de comorbidades que afetam principalmente os idosos, como a Osteoporose. A Osteoporose representa um problema de saúde pública, pois aumenta o risco de fratura de quadril nos idosos. As fraturas osteoporóticas geralmente estão relacionadas a quedas e possuem fatores de risco conhecidos, como idade avançada, sexo feminino, menopausa precoce, sedentarismo, entre outros. (PINHEIRO, 2010)

As publicações sobre o impacto econômico das fraturas de quadril em idosos no Brasil ainda não são muito numerosas. Oliveira et al (2017) conduziram um estudo sobre a epidemiologia e custos das fraturas de quadril em idosos no estado do Paraná. Nesse estudo, foram registradas 11.226 fraturas, sendo $66,8 \%$ em mulheres e $33,2 \%$ em homens. A mortalidade durante a internação foi de 5,9\%, maior no sexo masculino, nos pacientes com idade acima de 80 anos e nos negros e asiáticos. O custo total foi de $R \$ 29.393 .442,78$ e o custo médio por internação foi de $R \$ 2.618,34$. (OLIVEIRA, 2017) 
Dentro desse cenário de crescimento do número de idosos, é esperado um aumento da ocorrência de fraturas e sobretudo, as de quadril por estarem mais relacionadas a quedas. Esse acréscimo da incidência de fraturas de quadril impactará mais ainda o custo do SUS com esse tipo de fratura, agravando a situação precária da saúde pública no Brasil.

Logo, esse trabalho se justifica pelo impacto econômico dos custos por hospitalizações em razão de fraturas de quadril em idosos. Assim o objetivo deste estudo é delinear o perfil de custos relacionados a fratura de quadril no Distrito Federal, enfatizando o crescimento acelerado em número de idosos no Brasil nos últimos anos o que poderá implicar mais internações por acometimento do quadril, mostrando a importância da prevenção desse tipo de fratura.

\section{Fundamentação Teórica:}

O processo de envelhecimento relaciona-se com uma série de mudanças físicas e mentais que modificam, diretamente, a qualidade de vida do idoso. Atualmente o envelhecimento saudável não se relaciona apenas com a ausência de doenças, mas também com a capacidade do idoso em desenvolver suas tarefas diárias e de realizar suas atividades básicas sozinho. (VALER et al, 2015)

São várias as comorbidades que podem tornar a vida do idoso menos produtiva, dentre as quais a osteoporose, a mais comum dentre as doenças ósseo metabólicas, recebe maior destaque. A osteoporose é uma doença predisponente a quedas, devida a fragilidade óssea, e consequentemente torna-se um fator de risco primário para a fraturas do osso, principalmente as fraturas de quadril, o que reflete diretamente no aumento da morbi-mortalidade. (ALVES et al, 2017)

O custo econômico introduzido por uma doença pode ser classificado em duas grandes categorias: os custos diretos e indiretos. Os diretos referem-se aos custos médicos e não-médicos relacionados ao tratamento, diagnóstico e reabilitação da doença e os indiretos referem-se à perda de produção e produtividade trazidos pela doença, como por exemplo aqueles gerados por limitações físicas ou psicológicas e que impactam nas atividades de vida diária do indivíduo. 
Pasco e colaboradores avaliaram o impacto das fraturas de quadril na vida diária de mulheres. Todas as pacientes com lesões no fêmur proximal foram hospitalizadas. Cerca de $75 \%$ das pacientes com fraturas dos membros inferiores permaneceram restritas ao domicílio, necessitando do auxílio de andadores ou caminhando apenas pequenas distâncias por várias semanas. Após seis meses, 19,6\% das pacientes com o fêmur proximal acometido ainda necessitavam de auxílio para a higiene pessoal. Após um ano, apenas metade das pacientes referiam a mesma mobilidade e mais da metade ainda necessitavam de cuidados especiais. (PASCO et al., 2005)

O custo tanto social como econômico da fratura de quadril cresce ainda mais devido ao fato de que, mesmo após um período de internação longo, as taxas de mortalidade permanecem altas, os cuidados médicos permanecem intensivos e requerem programas de reabilitação por longo tempo. (ARNDT, 2009)

Essa mortalidade por fratura de quadril e os dias de trabalho perdidos pelos familiares dos pacientes durante o período de internação hospitalar indicam a grandeza dos custos indiretos impostos pela fratura. Pode-se perceber que a magnitude econômica e social de uma fratura de quadril em idosos não fica restrito ao paciente, mas também a família, aumentando assim a cadeia de custos tanto diretos como indiretos.

Torres e Ureña avaliaram os custos diretos com as fraturas de quadril na América Latina entre 1980 a 2003 , e variaram de US $\$ 4.500$ a US $\$ 6.000$ por paciente. O impacto econômico desse tipo de fratura variou entre os países avaliados, segundo diferenças populacionais e recursos de saúde disponíveis, sendo estimado em US\$ 5.500 por paciente para o Brasil. (MORALES-TORRES; GUTIÉRREZ-UREÑA, 2004)

\section{Metodologia:}

Trata-se de estudo descritivo, de base populacional, em série temporal, a partir de dados obtidos no Sistema de Informação Hospitalares do sistema único de saúde (SHI-SUS). O SHI-SUS faz parte de um conjunto de sistemas do departamento de informática do SUS, o DATASUS, que tem como objetivo disponibilizar informações que servem para análises mais fidedignas da situação sanitária da população. $\mathrm{O} \mathrm{SIH}$ - 
SUS registra todos os atendimentos provenientes de internações hospitalares, gerando relatórios para os gestores, o que possibilita os pagamentos dos estabelecimentos de saúde.

Foram coletados dados de morbidade hospitalar relativos à fratura de fêmur em pacientes com idade maior ou igual a 60 anos, representadas pelo código CID-10 S72 no período de 2008 - 2016 no Brasil e no Distrito Federal. Aplicou-se o uso de estatística analítica para projeção do custo total em 10 anos.

\section{Resultados e discussão:}

No Brasil foram registradas 367.321 internações o que representou $1,6 \%$ de todas as internações. O custo total das internações em 9 anos foi de $R \$ 810.774 .576$ milhões (mi), com média de $\mathrm{R} \$ 90 \mathrm{mi} / \mathrm{ano}$. A análise dos custos revela um padrão ascendente, mostrando aumento de $120 \%$ no período 2008 - 2016 e previsão de aumento em $74 \%$ no período 2016-2026 com gasto total cumulativo de 2,5 bilhões de reais no período total 2008-2026. O valor médio da cada internação foi de $R \$ 2.207,26$ ( $R \$ 2.062,90$ $R \$ 2.660,26)$. O gênero feminino foi responsável por $68 \%$ de todas as internações, com um custo de $\mathrm{R} \$ 553 \mathrm{mi}$. Quarenta e oito por cento do custo total incidiu sobre a faixa etária entre os maiores de 80 anos.

Já no Distrito Federal ocorreram 3.721 internações por fratura de quadril em idosos no período de 2008 a 2016, o que corresponde a cerca de $1 \%$ das internações por fratura de quadril no Brasil. Essas internações totalizaram um custo de $R \$$ $8.180 .600,70 \mathrm{mi}$, com média de $\mathrm{R} \$ 908.955,63$ por ano. Assim como no Brasil, os custos exibiram um padrão ascendente, com um aumento de 42\% entre 2008 a 2016. 


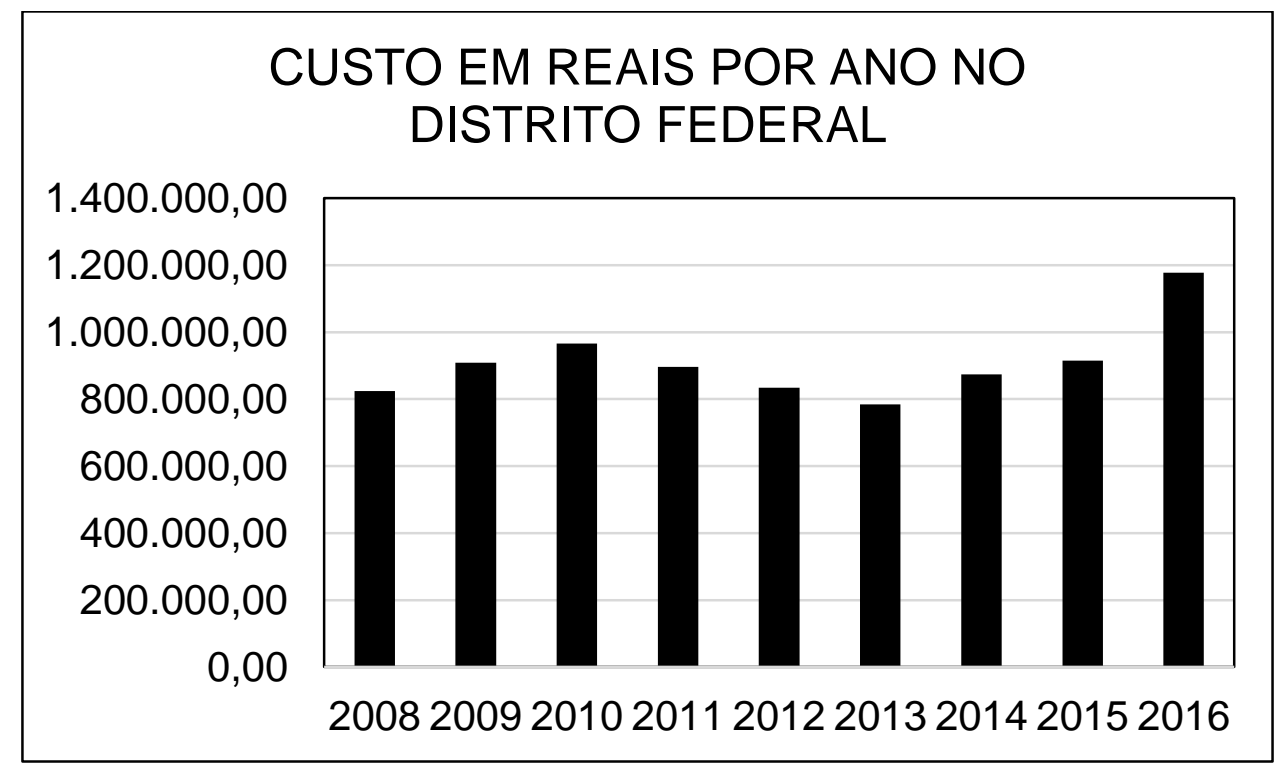

O aumento dos custos por fratura de quadril no Distrito Federal está relacionado com o processo de envelhecimento da população. O envelhecimento populacional iniciouse no final do século XIX na Europa e no século passado, estendeu-se por vários países do chamado terceiro mundo, dentre eles o Brasil. No caso brasileiro, a partir da década de 60, iniciou-se uma rápida e generalizada queda da taxa de fecundidade e consequentemente, um processo de envelhecimento. Segundo o Instituto Brasileiro de Geografia e Estatística (IBGE), a expectativa de vida em 2009 chegou a 73 anos e a proporção de idosos aumentou de 9,1\% em 1999 para 11,3\% em 2009. (MINAYO, 2012)

Cada internação teve um valor médio de $R \$ 2.198,50$. A média de dias internados foi de 18,2. Do total de número de internações, 2.428 foram do sexo feminino, o que representa $65 \%$ de todas as internações, com um custo de $\mathrm{R} \$ 5.428 .512,20 \mathrm{mi}$. 


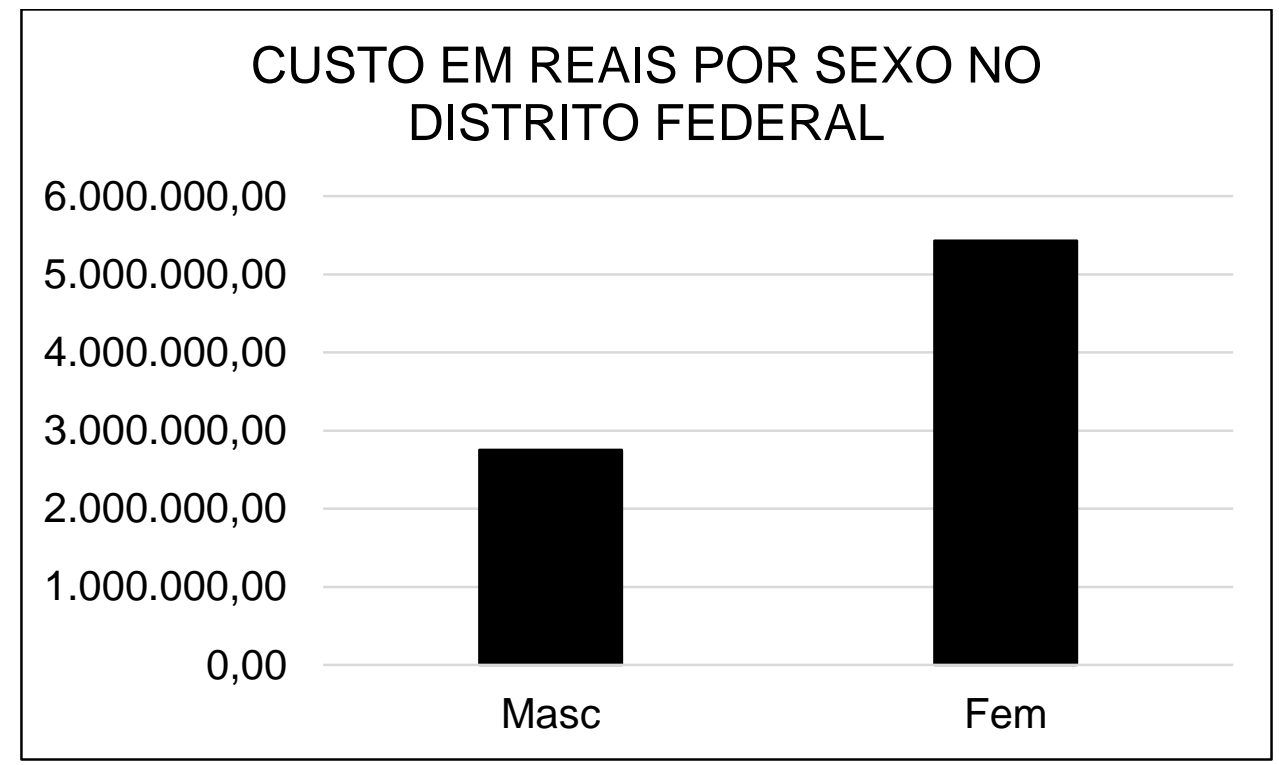

Essas fraturas são mais comuns na população feminina devido à maior propensão que mulheres possuem para osteoporose principalmente após a menopausa. Isso se explica pelo fato de que o estrogênio, hormônio reprodutivo feminino, afeta enorme quantidade de funções, incluindo o metabolismo ósseo e mineral. (SAMPAIO, 2011)

Em relação as faixas etárias, 891 pacientes tinham idade entre 60 a 69 anos, o que representa cerca de $24 \%$ do total de pacientes, 1.302 internados possuíam entre 70 a 79 anos, o que corresponde em porcentagem a 35\%. A faixa etária acima de 80 anos foi responsável pelo maior número de internações, 1.528 do total, representando $41 \%$ das 3.721 internações.

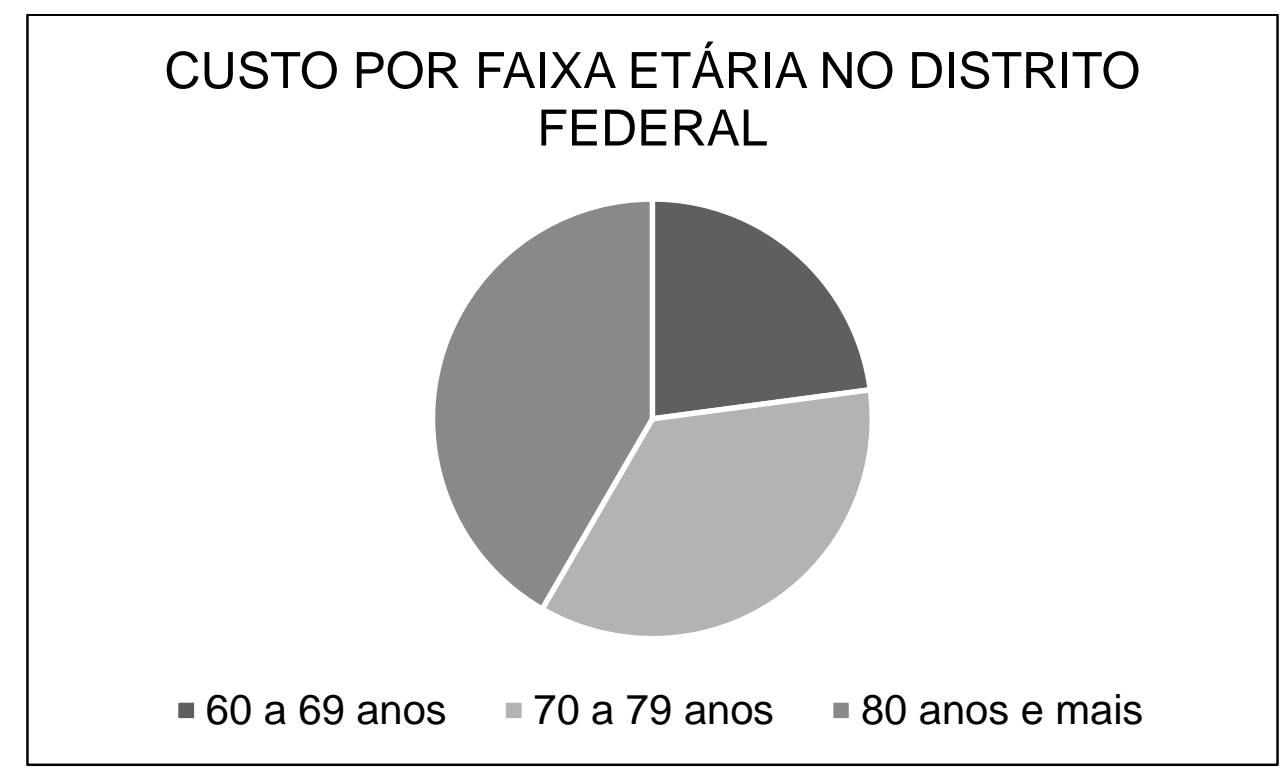


De acordo com as projeções das Nações Unidas, em 2025 o Brasil deverá contar com $33,6 \%$ de pessoas com mais de 45 anos de idade. (MINAYO, 2012) A prevalência de doenças crônicas não degenerativas nessa faixa etária, a relação entre envelhecimento, fragilidade óssea e fraturas impactam nos custos em saúde da população brasileira. As fraturas muitas vezes estão relacionadas com a osteoporose, doença diretamente relacionada com o aumento da idade.

A osteoporose é definida como uma doença do esqueleto caracterizada pelo comprometimento da resistência e da qualidade óssea, predispondo a aumento do risco de fraturas. (GUARNIEIRO, 2004) Osteoporose por ser uma doença assintomática, é geralmente diagnosticada tardiamente, muitas vezes já em decorrência de uma fratura por queda, como a fratura de quadril.

A fratura de fêmur está entre as lesões traumáticas mais comuns na população idosa, podendo ocorrer na região proximal, distal ou ainda na diáfise femoral. Esta fratura no idoso tem aumentado nas últimas décadas, sendo causada geralmente por traumas pequenos e não intencionais, como as quedas da própria altura, que ocorrem em função da senescência ou de fatores extrínsecos. Esse aumento pode ser notado pelo número de internações decorrentes de fraturas do quadril que representaram $1,6 \%$ de todas as internações no Brasil. (MUNIZ et al., 2007).

O presente estudo avaliou os custos de internação relacionados a fratura de quadril em pacientes idosos no Brasil. Os resultados mostraram uma média de 90 milhões de reais por ano com internações devido a esse tipo de fratura em pacientes acima de 60 anos, além de uma previsão de crescimento em $74 \%$ nos próximos 10 anos.

\section{Considerações finais:}

Os dados levantados a partir do estudo revelaram aumento da fratura de quadril em idosos, principalmente em mulheres, nos últimos anos. Esse evento merece atenção das autoridades sanitárias brasileiras pelo seu evidente impacto na saúde dos idosos e pelos consequentes prejuízos individuais e governamentais. A fratura de quadril requer cuidados hospitalares de média a longa duração, o que colabora para o aumenta substancialmente de custos diretos e indiretos, além de estar associada a 
riscos elevados de morbi-mortalidade e re-hospitalização. Altas taxas de mortalidade são em parte relacionadas com as comorbidades prévias dos pacientes, como osteoporose, e em parte, devido, direta ou indiretamente, ao evento em si da fratura de quadril.

Diante do exposto observa-se a importância de delinear o perfil e as estatísticas de hospitalizações de idosos e assim realizar promoção de saúde e prevenção de agravos direcionadas a diminuir a ocorrência de fraturas de quadril o que culminaria na redução dos custos diretos e indiretos.

\section{Referências:}

Anrdt, A.B.M. et al. $O$ custo direto da fratura de fêmur por quedas em pessoas idosas: análise no setor privado de saúde na cidade de Brasília. Revista Brasileira de Geriatria e Gerontologia. Universidade do Estado do Rio Janeiro, v. 14, n. 2, p. 221-231, 2011.

Guarnieiro, R. el al. Osteoporose: atualização no diagnóstico e princípios básicos para o tratamento. Revista Brasileira de Ortopedia. Vol. 39, No 9 - Setembro, 2004

Minayo, M.C.S. $O$ envelhecimento da população brasileira e os desafios para o setor saúde. Caderno de Saúde Pública, Rio de Janeiro, 28(2):208-209, 2012.

Muniz, C.F. Caracterização dos idosos com fratura de fêmur proximal atendidos em hospital escola público. Revista Espaço para a Saúde, Londrina, v.8, n.2, p.3338, jun.2007.

Morales Torres, J.; Gutiérrez Ureña, S. The burden of osteoporosis in Latin America. Osteoporosis int., v. 15, n. 8, p. 625-32, 2004.

Oliveira, C.C. et al. Epidemiologia das Fraturas fêmuras em Idosos e Custos para o estado do Paraná, Brasil. Acta ortop. bras. vol.25 no.4 São Paulo julho / ago. 2017. 
Pasco, J. A. et al. The human cost of fracture. Osteoporosis int., v. 16, n. 12, p. 2046$52,2005$.

Pinheiro MM, et al. O impacto da osteoporose no Brasil: Estudo Regional das Mulheres em Homens e Mulheres - O Estudo Brasileiro da Osteoporose (BRAZOS). Revista Brasileira de Reumatologia. 2010; 50 (2): 113-27.

Sampaio, P.R.L. A Osteoporose e a Mulher Envelhecida: fatores de risco. Revista Brasileira de Geriatria e Gerontologia, Rio de Janeiro, 2011.

Valer, D.B. et al. O significado de envelhecimento saudável para pessoas idosas vinculadas a grupos educativos. Revista Brasileira de Geriatria e Gerontologia, Rio de Janeiro, 2015. 